\title{
Diffusion tensor imaging to determine the potential motor network connectivity between the involved and non-involved hemispheres in stroke
}

\author{
Min-Hee Lee ${ }^{\mathrm{a}}$, Yong-Il Shin ${ }^{\mathrm{b}}$, Sang Hyeon Lee ${ }^{\mathrm{a}}$, Young Joo Cha ${ }^{\mathrm{d}}$, Dong Youn Kimª ${ }^{\mathrm{a}}$, Bong \\ Soo Han ${ }^{\mathrm{c}, *}$ and Sung H. You, ${ }^{\mathrm{d}, *}$ \\ ${ }^{a}$ Department of Biomedical Engineering, Yonsei University, 1 Yonseidae-gil, Wonju, Republic of South \\ Korea \\ ${ }^{b}$ Department of Rehabilitation Medicine, Pusan National University School of Medicine, Research \\ Institute for Convergence of Biomedical Science and Technology, Pusan National University Yangsan \\ Hospital, Pusan, Korea \\ ${ }^{c}$ Department of Radiological Science, Yonsei University, 1 Yonseidae-gil, Wonju, Republic of South \\ Korea \\ ${ }^{d}$ Movement Healing Lab, Department of Physical Therapy Program, Yonsei University, 1 Yonseidae- \\ gil, Wonju, Republic of South Korea
}

\begin{abstract}
Hemiparetic stroke is a common motor network disorder that affects a wide range of functional movements due to cortical and subcortical network lesions in stroke patients. Conventional magnetic resonance imaging (MRI) has been used to examine structural brain damage, but the integrity and connectivity of the whole brain are poorly understood. Hence, advanced neuroimaging with diffusion tensor imaging (DTI) has been developed to better localize fiber architecture and connectivity in the motor network or pathways that are responsible for motor impairments in hemiparetic stroke. To ascertain motor network connectivity between the involved and non-involved hemispheres in stroke patients, we analyzed the DTI data from all right hemiparetic stroke patients using fractional anisotropy (FA) and network parameters, including node degree and edge betweenness centrality (EBC). The FA values were substantially lower in the left hemisphere than the right hemisphere. Similarly, the node degree and EBC were significantly lower in the left hemisphere than the right hemisphere. The present brain network analysis may provide a useful neuropathway marker for accurate diagnosis and therapeutic intervention.
\end{abstract}

Keywords: Cerebrovascular accident, diffusion tensor imaging, brain network, fractional anisotropy

\footnotetext{
${ }^{*}$ Address for correspondence: Bong Soo Han, Department of Radiological Science, Yonsei University, 1 Yonseidae-gi 1, Wonju, Republic of South Korea. Tel.: +82-33-760-2381; Fax: +82-33-760-2562; E-mail: bshan@yonsei.ac.kr.

Sung H. You, Movement Healing Lab, Department of Physical Therapy Program, Yonsei University, 1 Yonseidae-g il, Wonju, Republic of South Korea. Tel.: +82-33-760-2476; Fax: +82-33-760-2496; E-mail: neurorehab@yonsei.ac.kr.
} 


\section{Introduction}

Hemiparetic stroke is a common and costly disorder resulting from cerebrovascular attack (CVA). Depending on the specific cortical or subcortical network lesions, it is often implicated with a wide range of important functional motor impairments such as walking and reaching [1,2]. MRI has been used to accurately diagnose cerebral lesions after CVA; however, the integrity and connectivity of the whole brain, which may represent important diagnostic and prognostic biomarkers for neurorehabilitation [3], have not been fully investigated. Hence, the neurophysiologic basis for the neurological deficits associated with CVA remains incompletely understood. Recently, DTI indices, such as mean diffusivity (MD), fractional anisotropy (FA), radial diffusivity (RD), and axial diffusivity (AD), have been used to localize the integrity of microstructural neural fibers of the sensorimotor pathways that are responsible for motor impairments in stroke [4-7]. In this study, we used only FA values because FA values were highly sensitive to microstructural changes [8]. However, DTI indices cannot provide information on the connectivity of distributed structural networks affecting motor function. The purpose of this preliminary case investigation was to determine microstructural changes and associated motor network connectivity in patients with unilateral hemiparetic stroke. Therefore, we applied a network analysis to DTI from hemiparetic stroke patients in order to investigate differences in network parameters, such as node degree and edge betweenness centrality, between the left and right motor-related regions (putamen, precentral gyrus, postcentral gyrus, and supplementary motor area). We hypothesized that intrahemispheric motor network disconnectivity would be associated with motor dysfunction in CVA.

\section{Materials and methods}

\subsection{Data acquisition and pre-processing}

A 3-Tesla MRI (Siemens, Germany) scanner was used to record DTI data from three patients with hemiparetic stroke resulting from left CVA. All patients had a wide range of lesions on their left hemispheres (Figure 1), and each patient's cortical or subcortical lesions are tabulated in Table 1. For DTI data acquisition, the standardized MRI parameters included repetition time (TR) $=6000 \mathrm{~ms}$, echo time $(\mathrm{TE})=105 \mathrm{~ms}$, and 20 non-collinear diffusion gradient directions at $\mathrm{b}=1000 \mathrm{~s} / \mathrm{mm}^{2}$ with $\mathrm{b}=0$ $\mathrm{s} / \mathrm{mm}^{2}$ image. We corrected distortions caused by eddy current and head motions, removed non-brain regions, and fitted the diffusion tensor model at each voxel using functional MRI of the brain (FMRIB) [9]. In brief, distortions caused by eddy current and head motions were corrected by applying 12parameter affine transformation to register each diffusion-weighted image to the b0 image obtained
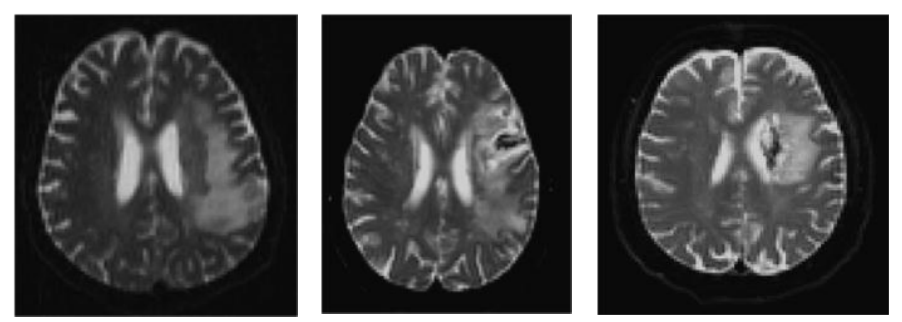

Fig. 1. Lesions on the left hemispheres of three patients. All patients had a wide range of lesions on their left hemispheres. The images are presented in a Radiological convention. 
Table 1

Affected cortical or subcortical regions of the left hemisphere for all patients

\begin{tabular}{ll}
\hline Patient & Affected cortical or subcortical regions of the left hemisphere \\
\hline Patient 1 & Frontal lobe, Postcentral gyrus, Precentral gyrus, Parietal lobe, Supramarginal gyrus, Temporal gyrus, Insula, \\
& Putamen \\
Patient 2 & Frontal lobe, Precentral gyrus, Angular gyrus, Supramarginal gyrus, Insula, Putamen \\
Patient 3 & Frontal lobe, Precentral gyrus, Caudate, Thalamus, Insula, Putamen \\
\hline
\end{tabular}

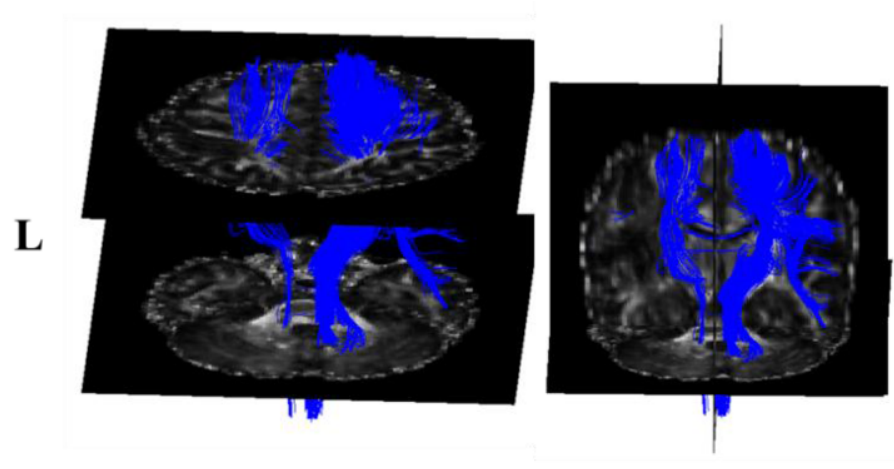

(a)

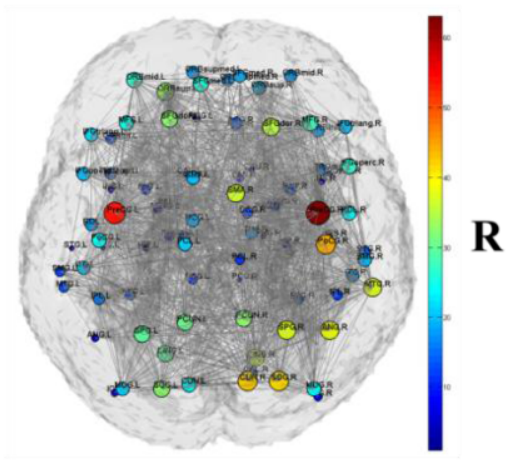

(b)

Fig. 2. The brain network construction derived from DTI data. (a) highlights DTI fiber tracts that terminate in motor cortex, including the precentral gyrus, postcentral gyrus, and supplementary motor areas whereas (b) illustrates network connectivity derived from DTI fiber tracking data.. The colors and circle sizes of each node are determined by the value of the node degree. The images are presented in a Neurology convention. Each node is located in the center of gravity of the AAL region. L, left; R, right.

without diffusion sensitizing gradient. DTI data of each subject were normalized to the Montreal Neurological Institute (MNI) space by using nonlinear tensor image registration algorithms [10]. Using the normalized DTI data, we performed fiber tracking using the Fiber Assignment by Continuous Tracking (FACT) algorithm in Dti-Studio (https://www.mristudio.org/) (Figure 2(a)) [11]. The regions of interest (ROIs) were defined as putamen, precentral gyrus, postcentral gyrus, and supplementary motor area and processed for data analysis because these regions primarily represent clinical motor network impairments in patients with hemiparetic stroke.

\subsection{Fractional anisotropy}

Fractional anisotropy (FA), defined in Eq. (1), is sensitive to fiber integrity in the brain:

$$
F A=\sqrt{\frac{3\left(\left(\lambda_{1}-\hat{\lambda}\right)^{2}+\left(\lambda_{2}-\hat{\lambda}\right)^{2}+\left(\lambda_{3}-\hat{\lambda}\right)^{2}\right)}{2\left(\lambda_{1}^{2}+\lambda_{2}^{2}+\lambda_{3}^{2}\right)}}
$$

where $\lambda_{1}, \lambda_{2}$, and $\lambda_{3}$ are eigenvalues and $\hat{\lambda}$ is the mean of the eigenvalues.

To compensate for loss of DTI image registration, FA maps were smoothed using a Gaussian kernel width of $8 \mathrm{~mm}$ (i.e., full width, half maximum) [12]. The FA value of gray matter in the brain is generally lower than 0.2 [13]. If the FA value is close to 0 in any brain region, it is defined as an 
isotropic lesion. We used a mean value of FA in each parcellated region to reveal the integrity of the whole brain.

\subsection{Network analysis}

\subsubsection{Network construction}

Nodes and edges are the basic elements of a network analysis. We defined the nodes to construct a brain network using an automated anatomical labeling (AAL) template already segmented into 90 cortical and subcortical regions [14]. Segmented regions in AAL template assigned indices from 1 to 90, each representing a node of the network. Two nodes were considered to be connected by an edge if two endpoints of tract were present one by one in each node. A binary undirected brain network was used by assigning a threshold that retains connectedness of over $90 \%$ for each network. Thus, if any two nodes $i$ and $j$ were connected, we assigned $A_{i j}=1$. If not, we assigned $A_{i j}=0$ in the adjacency matrix $A$. An example of the brain network construction is shown in Figure 2(b).

\subsubsection{Node degree}

To determine the integrity of the structural connectivity of the network regions, the node degree was computed at each node. The node degree is defined as the sum of the rows or columns in the adjacency matrix, ranging from 0 to 89 degrees [15]. A zero node degree represents virtually no associated connections between nodes, whereas an 89 node degree represents maximal possible connections in the network, indicating the extent of connectivity [15].

\subsubsection{Edge betweenness centrality}

The edge betweenness centrality (EBC) is defined as the number of the shortest neural pathways transmitting to other associated edges in the network [16]. EBC can determine the relative portal pathway of edges and identify key edges, providing important information about the centralized communication portal edges or sites between other involved nodes in the network [16]. An edge $e$ in the EBC is defined as the fraction of shortest paths between any two adjacent nodes $i$ and $j$ that pass through an edge.

$$
E B C(e)=\sum_{i, j \in N, i \neq j} \frac{\sigma_{i j}(e)}{\sigma_{i j}}
$$

where $N$ is the set of nodes, $\sigma_{i j}$ represents the number of shortest neural pathways between nodes $i$ and $j$, and $\sigma_{i j}(e)$ denotes the number of shortest paths between nodes $i$ and $j$ that pass through the edge. A zero EBC indicates the absence of connections between nodes. Edges with a high EBC are considered 'bridges' or portal neural pathways in the network [17].

\section{Results}

The measured FA values, node degree and EBC for the patients were tabulated in Tables 2 and 3, respectively. As shown in Table 2, FA in the putamen and supplementary motor areas was reduced in the left (involved) hemisphere compared to the right (non-involved) hemisphere. Similarly, node degree and EBC were lower in the left (involved) than the right (non-involved) hemisphere (Figure 3). 
Table 2

Comparisons of fractional anisotropy and node degree data between the right and left hemispheres

\begin{tabular}{|c|c|c|c|c|c|c|}
\hline \multicolumn{7}{|c|}{ Fractional anisotropy in the regions of interest (ROIs) } \\
\hline & Patient 1 & & Patient 2 & & Patient 3 & \\
\hline & $\begin{array}{l}\text { Right } \\
\text { hemisphere }\end{array}$ & $\begin{array}{l}\text { Left } \\
\text { hemisphere }\end{array}$ & $\begin{array}{l}\text { Right } \\
\text { hemisphere }\end{array}$ & $\begin{array}{l}\text { Left } \\
\text { hemisphere }\end{array}$ & $\begin{array}{l}\text { Right } \\
\text { hemisphere }\end{array}$ & $\begin{array}{l}\text { Left } \\
\text { hemisphere }\end{array}$ \\
\hline Putamen & 0.2053 & 0.1685 & 0.2028 & 0.1745 & 0.2016 & 0.1923 \\
\hline Supplementary motor area & 0.1733 & 0.1576 & 0.1597 & 0.1368 & 0.1478 & 0.1312 \\
\hline \multicolumn{7}{|l|}{ Node degree in ROIs } \\
\hline & Patient 1 & & Patient 2 & & Patient 3 & \\
\hline & $\begin{array}{l}\text { Right } \\
\text { hemisphere }\end{array}$ & $\begin{array}{l}\text { Left } \\
\text { hemisphere }\end{array}$ & $\begin{array}{l}\text { Right } \\
\text { hemisphere }\end{array}$ & $\begin{array}{l}\text { Left } \\
\text { hemisphere }\end{array}$ & $\begin{array}{l}\text { Right } \\
\text { hemisphere }\end{array}$ & $\begin{array}{l}\text { Left } \\
\text { hemisphere }\end{array}$ \\
\hline Precentral gyrus & 74 & 57 & 85 & 63 & 71 & 68 \\
\hline Postcentral gyrus & 52 & 24 & 29 & 38 & 43 & 38 \\
\hline Supplementary motor area & 38 & 20 & 28 & 29 & 54 & 47 \\
\hline
\end{tabular}

Table 3

Comparisons of edge betweenness centrality between the right and left hemispheres

\begin{tabular}{lllllll}
\hline Edge betweenness centrality & & & & \\
\hline Connections in ROIs & Patient 1 & & Patient 2 & & Patient 3 & \\
& $\begin{array}{l}\text { Right } \\
\text { hemisphere }\end{array}$ & $\begin{array}{l}\text { Left } \\
\text { hemisphere }\end{array}$ & $\begin{array}{l}\text { Right } \\
\text { hemisphere }\end{array}$ & $\begin{array}{l}\text { Left } \\
\text { hemisphere }\end{array}$ & $\begin{array}{l}\text { Right } \\
\text { hemisphere }\end{array}$ & $\begin{array}{l}\text { Left } \\
\text { hemisphere }\end{array}$ \\
\hline ANG - PreCG & 25.4263 & 0 & 43.5863 & 0 & 15.4872 & 7.6938 \\
HES - PoCG & 49.5991 & 0 & 0 & 0 & 22.0472 & 0 \\
CAU - PreCG & 68.5009 & 0 & 0 & 0 & 0 & 0 \\
\hline
\end{tabular}

Note: PreCG, precentral gyrus; CAU, caudate; PoCG, postcentral gyrus; ANG, angular gyrus; HES, transverse temporal gyrus.
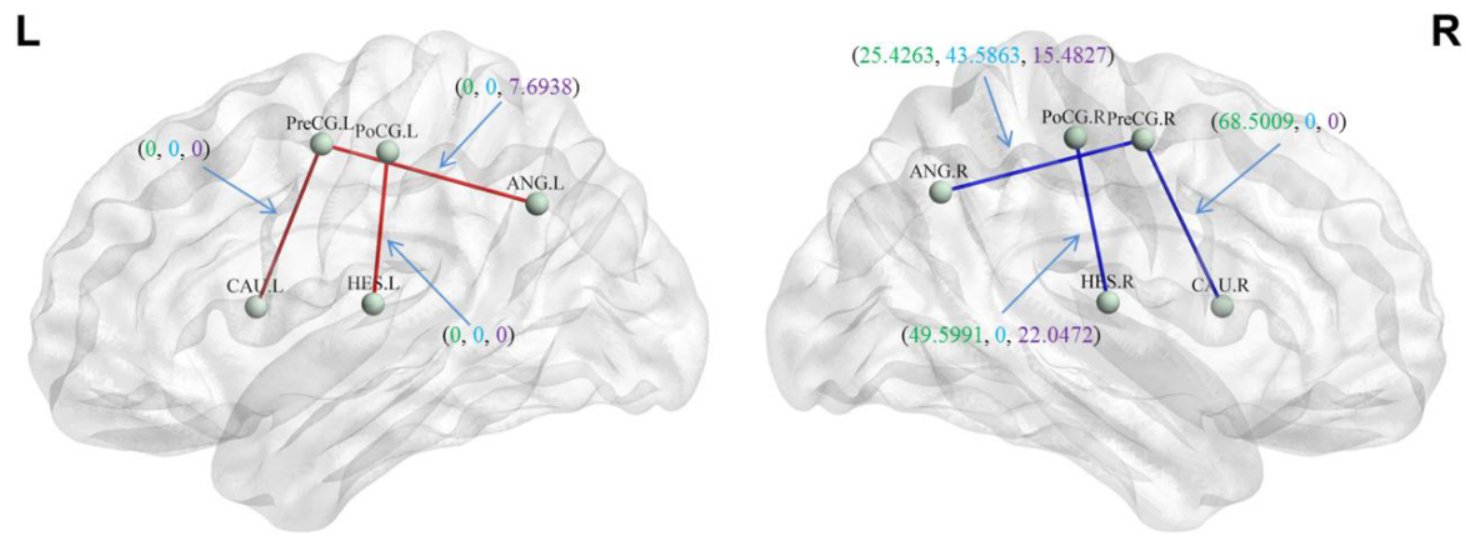

Fig. 3. The neural motor pathway connectivity between the right and left hemispheres is highlighted. Edge betweenness centrality values demonstrate the relative difference in motor pathway connectivity between the right and left hemispheres. The images are presented in a Neurology convention. PreCG, precentral gyrus; CAU, caudate; PoCG, postcentral gyrus; ANG, angular gyrus; HES, transverse temporal gyrus; R, right; L, left.

\section{Discussion}

The present investigation used advanced neuroimaging analysis with DTI to determine the potential motor network connectivity between the involved and non-involved hemispheres in stroke patients 
[18]. Most importantly, we demonstrated that the FA values were substantially lower in the left hemisphere than the right hemisphere. Similarly, the node degree and edge betweenness centrality network parameters were significantly lower in the left hemisphere than the right hemisphere, indicating impaired motor network connectivity in the left hemisphere in stroke patients. Moreover, the motor network disruption observed in DTI was closely associated with MRI markers and clinical motor dysfunction measures, such as spasticity, paresis, and hemiparetic gait.

Conventionally, MRI has been used to identify neuropathology in the whole brain after CVA, which partly accounts for the distribution of underlying motor pathway lesions [19]. Nevertheless, an advanced DTI imaging method can help reveal impaired motor network connections characterized by involvement of precentral and postcentral intrahemispheric connectivity that would not have been identified by conventional MRI techniques [20]. This impairment of the connectivity of pre- and postcentral areas provides a neuropathological mechanism for motor deficits observed in CVA.

The present study has limitations. We used the unaffected hemisphere as control or reference for our novel network computational analysis because our T2 weighted MR images (b0 images) showed relatively intact diagnostic image in the unaffected hemisphere. However, this preliminary investigation invites a future study to compare normal and pathological motor network analysis with a larger sample size. Additionally, a combination of fMRI and DTI would certainly provide more comprehensive structural and functional motor network information. Hence, in future studies, a combination of resting-state fMRI and DTI analysis should be considered to better understand neural function, structural substrates and network connectivity in normal and neurological populations.

Certainly, our DTI findings of altered motor network measures in CVA, and their associations with motor function, corroborate previous studies in CVA and associated motor [21, 22] and cognitive impairments [20], which have been identified as white matter fiber lesions. The consistency in previous studies of white matter fiber pathology supports clinical ramifications of microstructural network integrity or connectivity for motor function and the suitability of DTI network measures as important neuropathway markers for motor dysfunction in CVA.

Taken together, this neuroimaging evidence highlights the importance of motor network disconnectivity as a mediating mechanism between white matter neuropathology and motor dysfunction in CVA. DTI network analysis may help assisting the clinicians when diagnosing motor network impairments in patients with hemiparetic stroke.

\section{Conclusion}

Advanced application of network analysis using diffusion tensor MRI was suitable for identification of white matter network intra- and interhemispheric connectivity, which was impaired in CVA. The degree of disruption was characterized by the impaired FA, node degree, and edge betweenness centrality network analysis parameters and was related to motor impairment. The present network analysis may provide a useful neuropathway marker for accurate diagnosis and therapeutic intervention.

\section{Acknowledgments}

This research was supported by the Leading Foreign Research Institute Recruitment Program through the National Research Foundation of Korea (NRF) funded by the Ministry of Science, ICT \& 
Future Planning (2010-00757).

\section{References}

[1] G.E. Gresham, P.W. Duncan and W.B. Stason, Post-Stroke Rehabilitation, DIANE Publishing, Rockville, MD, 1997.

[2] F. Alarcon, J.C.M. Zijlmans, G. Duenas and N. Cevallos, Post-stroke movement disorders: Report of 56 patients, Journal of Neurology, Neurosurgery \& Psychiatry 75 (2004), 1568-1574.

[3] P.J. Basser, J. Mattiello and D. LeBihan, MR diffusion tensor spectroscopy and imaging, Biophysical Journal 66 (1994), 259-267.

[4] S.L. Heller, L.A. Heier, R. Watts, T.H. Schwartz, N. Zelenko, W. Doyle and O. Devinsky, Evidence of cerebral reorganization following perinatal stroke demonstrated with fMRI and DTI tractography, Clinical Imaging 29 (2005), $283-287$.

[5] D. Le Bihan, J.F. Mangin, C. Poupon, C.A. Clark, S. Pappata, N. Molko and H. Chabriat, Diffusion tensor imaging: Concepts and applications, Journal of Magnetic Resonance Imaging 13 (2001), 534-546.

[6] P.C. Sundgren, Q. Dong, D. Gomez-Hassan, S.K. Mukherji, P. Maly and R. Welsh, Diffusion tensor imaging of the brain: Review of clinical applications, Neuroradiology 46 (2004), 339-350.

[7] P.J. Basser and C. Pierpaoli, Microstructural and physiological features of tissues elucidated by quantitative-diffusiontensor MRI, Journal of Magnetic Resonance 213 (2011), 560-570.

[8] A.L. Alexander, J.E. Lee, M. Lazar and A.S. Field, Diffusion tensor imaging of the brain, Neurotherapeutics 4 (2007), 316-329.

[9] M. Jenkinson, C.F. Beckmann, T.E. Behrens, M.W. Woolrich and S.M. Smith, Fsl, Neuroimage 62 (2012), 782-790.

[10] H. Zhang, B.B. Avants, P.A. Yushkevich, J.H. Woo, S. WANG, L.F. McCluskey, L.B. Elman, E.R. Melhem and J.C. Gee, High-dimensional spatial normalization of diffusion tensor images improves the detection of white matter differences: An example study using amyotrophic lateral sclerosis, IEEE Transactions on Medical Imaging 26 (2007), 1585-1597.

[11] S. Mori, B.J. Crain, V. Chacko and P. Van Zijl, Three-dimensional tracking of axonal projections in the brain by magnetic resonance imaging, Annals of Neurology 45 (1999), 265-269.

[12] J.E. Lee, M.K. Chung, M. Lazar, M.B. DuBray, J. Kim, E.D. Bigler, J.E. Lainhart and A.L. Alexander, A study of diffusion tensor imaging by tissue-specific, smoothing-compensated voxel-based analysis, Neuroimage 44 (2009), 870883.

[13] D. Qiu, L.H. Tan, K. Zhou and P.L. Khong, Diffusion tensor imaging of normal white matter maturation from late childhood to young adulthood: Voxel-wise evaluation of mean diffusivity, fractional anisotropy, radial and axial diffusivities, and correlation with reading development, Neuroimage 41 (2008), 223-232.

[14] N. Tzourio-Mazoyer, B. Landeau, D. Papathanassiou, F. Crivello, O. Etard, N. Delcroix, B. Mazoyer and M. Joliot, Automated anatomical labeling of activations in SPM using a macroscopic anatomical parcellation of the MNI MRI single-subject brain, Neuroimage 15 (2002), 273-289.

[15] E. Bullmore and O. Sporns, Complex brain networks: Graph theoretical analysis of structural and functional systems, Nature Reviews Neuroscience 10 (2009), 186-198.

[16] M. Girvan and M.E. Newman, Community structure in social and biological networks, Proceedings of the National Academy of Sciences 99 (2002), 7821-7826

[17] G. Gong, Y. He, L. Concha, C. Lebel, D. W. Gross, A.C. Evans and C. Beauleu, Mapping anatomical connectivity patterns of human cerebral cortex using in vivo diffusion tensor imaging tractography, Cerebral Cortex 19 (2009), 524536.

[18] M. Urbanski, M.T. De Schotten, S. Rodrigo, C. Oppenheim, E. Touzé, J.F. Méder, K. Moreau, C. Loeper-Jeny, B. Dubois and P. Bartolomeo, DTI-MR tractography of white matter damage in stroke patients with neglect, Experimental Brain Research 208 (2011), 491-505.

[19] Y. Cao, L. D'Olhaberriague, E.M. Vikingstad, S.R. Levine and K.M. Welch, Pilot study of functional MRI to assess cerebral activation of motor function after poststroke hemiparesis, Stroke 29 (1998), 112-122.

[20] A.J. Lawrence, A.W. Chung, R.G. Morris, H.S. Markus and T.R. Barrick, Structural network efficiency is associated with cognitive impairment in small-vessel disease, Neurology 83 (2014), 304-311.

[21] S.H. Jang, S.H. You, Y.H. Kwon, M. Hallett, M.Y. Lee and M.Y. Ahn, Cortical reorganization associated lower extremity motor recovery as evidenced by functional MRI and diffusion tensor tractography in a stroke patient, Restorative Neurology and Neuroscience 23 (2005), 325-329.

[22] S.H. Jang, Y.H. Kim, Y.H. Kwon, S.H. You, B.S. Han and W.M. Byun, Restoration of the corticospinal tract compressed by hematoma: A tractography study using diffusion tensor imaging, Archives of Neurology 63 (2006), 140-141. 